\title{
Laser therapy for dentine hypersensitivity
}

\section{Abstracted from}

\section{He S, Wang Y, Li X, Hu D.}

Effectiveness of laser therapy and topical desensitising agents in treating dentine hypersensitivity: a systematic review. J Oral Rehabil 2011; 38: 348-358.

Address for correspondence: Dr. Xue Li, Department of Preventive Dentistry, West China College of Stomatology, Sichuan University, No. 14, Section 3, Road Renminnan, Wuhou District, Chengdu, China. E-mail: gardonlixue@sina.com

\section{Question: In patients with dentine hypersensitivity is laser therapy more effective than other desensitising agents?}

Data sources Medline, Embase, the Cochrane Central database as well as the Cochrane Oral Health Group's Trials Register and the National Research Register. In addition relevant journals were hand searched from 2000 to 2010 (Lasers in Medical Sciences, Lasers in Surgery and Medicine, Photomedicine and Laser Surgery, Photodiagnosis and Photodynamic Therapy, Journal of Oral Rehabilitation, Journal of Periodontology, Journal of Clinical Periodontology, Journal of Endodontics, Clinical Oral Investigations, Journal of Dental Research, Journal of Oral Laser Applications, Journal of Periodontal Research and Periodontology 2000) together with the reference lists of relevant trials.

Study selection Randomised controlled trials (RCT) that included patients with two or more hypersensitive teeth confirmed by evaporative stimulus or tactile hypersensitivity assessment, comparing laser therapy versus other topical desensitising agents, such as fluoride varnish, dentine bonding agents etc, that were published in English. Data extraction and synthesis Studies were assessed for quality by two reviewers independently and data were extracted using a standardised form. Because of heterogeneity of the studies meta-analysis was not performed, so a qualitative synthesis is presented.

Results Eight trials (234 participants) met the inclusion criteria. Half of the included studies compared GaALAS laser with topical desensitising agents, but the findings were conflicting. The remaining studies involved Nd:YAG laser, Er:YAG laser and CO2 laser, and all showed that the three types of lasers were superior to topical desensitising agents, but the superiority was slight.

Conclusions The review suggests that laser therapy has a slight clinical advantage over topical medicaments in the treatment of dentine hypersensitivity. However more large sample-sized, long-term, highquality randomised controlled clinical trials are needed before definitive conclusions can be made.

\section{Commentary}

Dentine hypersensitivity, a short, sharp pain arising from exposed dentine in response to stimuli that cannot be ascribed to any other form of dental defect or pathology, is a common presentation in the dental office. General dentists reported to frequently use on average three products or techniques and to have tried eight different products or techniques for the treatment or management of this condition. The plethora of products and techniques may indicate that no single treatment is effective and, currently, there is no evidence demonstrating the superiority of any one desensitising agent. The reviewed study assessed the effectiveness and safety of lasers in the treatment of dentine hypersensitivity through a systematic review.

Lasers have been used for this purpose since the mid-1980s and their effectiveness has been tested in just a few randomised clinical trials. This systematic review identified eight randomised clinical trials with moderate- to high-risk of bias and concluded that lasers are slightly superior to other topical desensitising treatments. In particular, Nd:YAG laser, Er:YAG laser and CO2 laser therapies were superior to topical desensitising agents (eg fluoride varnish), while results for GaALAS laser were inconsistent.

The systematic review compared laser treatments to an active control group - topical desensitising agents. The reason for choosing this comparison group instead of a placebo is not clear. The testing of effectiveness of dentine hypersensitivity treatments has been challenged by the difficulty in proving a result superior to the placebo effect. In contrast with the current systematic review, another systematic review on this topic, including only placebo controlled trials, identified three randomised controlled trials which showed that lasers were not superior to placebo.

A descriptive sysnthesis of the trials is provided in the systematic review; meta-analysis was not considered appropriate by the authors given the heterogeneity of the included studies. A random-effects meta-analysis could have been performed to calculate standardised mean differences which would take into account the different scales used in the trials. In addition, statistical heterogeneity could have been reported.

Based on the data from the included studies, a meta-analysis would yield the following results: Er:YAG laser ( $\mathrm{n}=2$ trials) and $\mathrm{CO} 2$ $(\mathrm{n}=1$ trial) were statistically significantly superior to other topical desensitising agents, while GaALAS laser ( $n=4$ trials) and Nd:YAG laser ( $\mathrm{n}=2$ trials) were not (Figure 1 ). The clinical heterogeneity and the quality of the trials and the high level of statistical heterogeneity (I2> 90\% for all comparisons, except Nd:YAG laser) would prevent us from relying on those summary estimates.

Safety of the laser treatments was one of the objectives of the systematic review. Five trials reported on adverse effects, and no detrimental pulpal effects, allergic reactions or clinically detectable complications during the follow-up periods were observed. The authors concluded that, based on the limited evidence, laser 
Heterogeneity: $\mathrm{Tau}^{2}=0.00 ; \mathrm{Chi}^{2}=61.09, \mathrm{df}=1(\mathrm{P}<0.00001) ; \mathrm{I}^{2}=95 \%$

Test for overall effect: $Z=0.08(P=0.94)$

\title{
1.1.2 Nd:YAG laser
}

$\begin{array}{lr}\text { Kara } 2009 & 0.43[-0.46,1.32] \\ \text { Kumar } 2005 & -0.21[-1.09,0.67] \\ \text { Subtotal }(95 \% \mathrm{Cl}) & \mathbf{0 . 1 1}[-0.52,0.74] \\ \text { Heterogeneity: } \mathrm{Tau}^{2}=0.00 ; \mathrm{Chi}^{2}=1.02, \mathrm{df}=1(\mathrm{P}=0.0005) ; \mathrm{I}^{2}=2 \% \\ \text { Test for overall effect: } \mathrm{Z}=3.59(\mathrm{P}=0.0003)\end{array}$

\subsubsection{ErYAG laser}

$\begin{array}{ll}\text { Ipci } 2009 & -1.86[-2.23,-1.50] \\ \text { Scharwz } 2002 & -3.28[-3.99,-2.57] \\ \text { Subtotal }(95 \% \mathrm{Cl}) & -2.54[-3.92,-1.15]\end{array}$

Heterogeneity: $\mathrm{Tau}^{2}=0.92 ; \mathrm{Chi}^{2}=12.14, \mathrm{df}=1(\mathrm{P}=0.0005) ; \mathrm{I}^{2}=92 \%$

Test for overall effect: $Z=3.59(P=0.0003)$

\author{
1.1.4 CO2 \\ Ipci 2009 \\ $-1.71[-2.06,-1.35]$ \\ Subtotal $(95 \% \mathrm{CI})$ \\ $-1.71[-2.06,-1.35]$ \\ Heterogeneity: Not applicable \\ Test for overall effect: $Z=9.44(P<0.00001)$
}

Total (95\% CI)

$-0.73[-1.71,0.25]$

Heterogeneity: $\mathrm{Tau}^{2}=2.14 ; \mathrm{Chi}^{2}=276.12, \mathrm{df}=8(\mathrm{P}<0.00001) \mathrm{I}^{2}=97 \%$

Test for overall effect: $Z=1.47(P=0.14)$

Test for subgroup differences: $\mathrm{Chi}^{2}=32.83, \mathrm{df}=3(\mathrm{P}<0.00001), \mathrm{I}^{2}=90.9 \%$

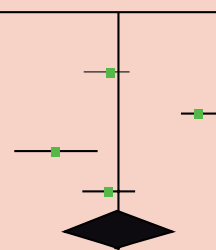

